\title{
RECENT RESULTS ON SEMIMUONIC DECAYS OF CHARM MESONS FROM FERMILAB EXPERIMENT E653
}

\section{B. GIBAUT}

Phyaic, Department, Virginia Polytechnic Institute and State University Blacksburg, VA 24061, U.S.A.

D. M. POTTER

Physicd Department, Carmegic Mellon University Pittsburgh, PA 15219, U.S.A.

\begin{abstract}
Fermilab experiment E653 has recently reported several results on the semimuonic decays of charm mesons. In this paper we review these results, which include the measurement of form factor ratios in $D^{+} \rightarrow \bar{K}^{0} \mu^{+} \nu$, the first measurement of form factor ratios in $D_{i}^{+} \rightarrow \phi \mu^{+} \nu$, the first observation of $D_{i}^{+} \rightarrow\left(\eta \mu^{+} \nu+\eta^{\prime} \mu^{+} \nu\right)$, and of the Cabibbo disfarored chanael $D^{+} \rightarrow \rho^{0} \mu^{+} \nu$, and an upper limit for rare $D^{0}$ decays.
\end{abstract}

\section{Introduction}

The semileptonic decays of charm mesons are dominated by three-body modes, which can be interpreted in terms of the spectator model ${ }^{a}$. All strong interaction effects, arising from the internal structure of the initial and final state hadrons, can be described by transition form factors. Semileptonic charm decays can thus provide a useful probe of hadronic structure in a regime where perturbative QCD cannot be applied. Quark models, lattice gauge calculations and QCD sum rules have been used to calculate the form factors, and the theoretical predictions can be compared to the measured values. Alternatively, the theoretical input can be combined with the experimental results to extract ratios of CKM matix elements and other quantities of interest. A potentially important example is the use of heavy quark effective theory ${ }^{2}$ to determine $\left|V_{u b}\right| /\left|V_{c d}\right|$ from measurements of the modes $B \rightarrow \rho l \nu$ and $D \rightarrow \rho l \nu$.

In this review we summarize the recent results of Fermilab experiment E653 for

asee reference 1 for a recent review of semileptonic charm decays. 
semimuonic charm meson decays, with particular emphasis on form factor measurements.

The E653 apparatus and analysis technique are described in reference 3. Using a $600 \mathrm{GeV} / \mathrm{c} \pi^{-}$beam incident on an emulsion target, and a trigger that required a penetrating muon, data corresponding to $2.5 \times 10^{8}$ interactions were recorded in a two-spectrometer detector. The upstream spectrometer consisted of an 18 plane silicon microstrip vertex detector and a wide aperture magnet followed by $55 \mathrm{drift}$ chamber planes. The muon track was remeasured and clearly identified by the downstream spectrometer, which consisted of a toroidal iron magnet and two sets of drift chambers. Neither photon identification nor hadron identification was used, and particle masses were assigned to tracks according to the decay mode under study.

\section{The Minimum Parent Mass}

Central to the E653 analysis of semimuonic charm decay is the minimum parent mass variable ${ }^{4}, \boldsymbol{M}_{\min }$, defined in terms of the invariant mass of the charged tracks originating from the parent charm meson decay vertex, $M_{v i s}$, and their momentum perpendicular to the parent direction, $p_{T}$ :

$$
M_{\min }=\sqrt{M_{\mathrm{vis}}^{2}+p_{T}^{2}}+\sqrt{m_{\nu}^{2}+p_{T}^{2}},
$$

where the mass of the neutrino, $m_{\nu}$, is taken to be zero. For a sample of decay vertices in which all charged daughter tracks have been assigned the correct masses and only the neutrino is undetected, the distribution of $M_{\min }$ has a distinctive cusp at the charm meson mass. Incorrect mass assignments or additional undetected neutral particles broaden and shift the peak. Consequently, $M_{\min }$ can be used to discriminate between decay channels in the absence of hadron identification and neutral particle detection.

\section{The Modes $D^{0} \rightarrow K^{-} \mu^{+} \nu$ and $D^{+} \rightarrow \bar{K}^{*}(892)^{0} \mu^{+} \nu$}

The variable $M_{\min }$ was used in selecting samples of $D^{0} \rightarrow K^{-} \mu^{+} \nu$ and $D^{+} \rightarrow$ $\bar{K}^{*}(892)^{0} \mu^{+} \nu$ decays from which the ratio $\Gamma\left(D \rightarrow K^{*} l \nu\right) / \Gamma(D \rightarrow K l \nu)$ was determined. Figure 1 shows the distribution in $M_{\min }$ for two-prong semimuonic decay vertices passing selection criteria designed to extract charm signals ${ }^{5}$. The event sample defined by $1.6<M_{\min }<2.0 \mathrm{GeV} / \mathrm{c}^{2}$ is dominated by the $D^{0} \rightarrow K^{-} \mu^{+} \nu$ mode, but contains background both from recontruction errors, and from other two-prong charm semimuonic modes. An estimate of the reconstruction background is given by the muonic two-prong vertices in which both tracks have the same charge; the distribution for such vertices is shown shaded in Figure 1. In order to estimate the physics background, and thus to determine the number of $D^{0} \rightarrow K^{-} \mu^{+} \nu$ decays in the final sample, the $M_{\min }$ distribution was simulated using the world average values ${ }^{6}$ for $D^{0} \rightarrow K^{-} \mu^{+} \nu$ and $D^{0} \rightarrow K^{*-} \mu^{+} \nu$, the measured rate ${ }^{7}$ for $D^{0} \rightarrow \pi^{-} \mu^{+} \nu$, and the ratio $\Gamma\left(D^{0} \rightarrow \rho^{-} \mu^{+} \nu\right) / \Gamma\left(D^{0} \rightarrow \pi^{-} \mu^{+} \nu\right)$ given by the ISGW 
quark model ${ }^{8}$. The result of the simulation is shown as the solid histogram in Figure 1. The $D^{0} \rightarrow K^{-} \mu^{+} \nu$ mode constituted $75 \%$ of the events within the indicated $M_{\min }$ cuts, and consequently, the sensitivity of the $D^{0} \rightarrow K^{-} \mu^{+} \nu$ yield to the branching ratios used in the simulation was small.

Figure 2a shows the $\boldsymbol{M}_{\min }$ distribution for three-prong semimuonic decay vertices and for simulated $D^{+} \rightarrow \bar{K}^{*}(892)^{0} \mu^{+} \nu, \bar{K}^{* 0} \rightarrow K^{-} \pi^{+}$decays; agreement is very good in the region of the peak, indicating that the $D^{+} \rightarrow \bar{K}^{* 0} \mu^{+} \nu$ mode dominates this event sample. The principal background is that due to recontruction errors, and was estimated by the number of muonic three-prong vertices in which the muon charge was opposite to the net charge of the vertex. The distribution in $M_{\min }$ for such "wrong-sign" vertices is shown shaded in Figure 2a. That the region of the $M_{\min }$ peak is indeed dominated by $D^{+} \rightarrow \bar{K}^{* 0} \mu^{+} \nu$ is confirmed by Figure $2 \mathrm{~b}$, which shows the distribution in $K \pi$ mass, $M_{K \pi}$, for vertices of Figure 2a with $1.6<M_{\min }<2.0 \mathrm{GeV} / \mathrm{c}^{2}$; the distribution for the wrong-sign vertices is shown shaded. A fit to the $M_{K \pi}$ spectrum, using a p-wave Breit-Wigner for the $K^{*}$ resonance and a non-resonant s-wave for the physics background, yielded a final sample of $D^{+} \rightarrow \bar{K}^{* 0} \mu^{+} \nu$ decays with less than $10 \%$ total background in the mass range $0.83 \leq M_{K \pi} \leq 0.95 \mathrm{GeV} / \mathrm{c}^{2}$.

Additional information is required to extract the ratio $\Gamma\left(D \rightarrow K^{*} l \nu\right) / \Gamma(D \rightarrow K l \nu)$ from the samples of $D^{0} \rightarrow K^{-} \mu^{+} \nu$ and $D^{+} \rightarrow \bar{K}^{*}(892)^{0} \mu^{+} \nu$ events; strong isospin is assumed to be conserved in semileptonic $D$ decays, but the cross section ratio, $\sigma\left(D^{0}\right) / \sigma\left(D^{+}\right)$, must be determined from the data. From the yield of events in the fully constrained all-charged modes $D^{+} \rightarrow K \pi \pi, D^{0} \rightarrow K \pi$ and $D^{0} \rightarrow K \pi \pi \pi$, $\sigma\left(D^{0}\right) / \sigma\left(D^{+}\right)$was found to be $1.65 \pm 0.31 \pm 0.31$, and the result

$$
\frac{\Gamma\left(D \rightarrow K^{*} l \nu\right)}{\Gamma(D \rightarrow K l \nu)}=0.43 \pm 0.09 \pm 0.09
$$

was obtained ${ }^{5}$. This result is consistent with the measurements of several other experiments, but a factor of two smaller than quark model predictions ${ }^{6}$. Since most models successfully predict the decay rate for $D \rightarrow K l \nu$, the discrepancy apparently lies in the decay mode involving a final state vector meson.

\section{Search for Rare Decay Modes}

The Voloshin-Shifman (VS) limit $^{9}$ implies that the semileptonic decay of mesons containing heavy quarks should be saturated by decay to the lowest mass pseudoscalar and vector mesons. Although not strictly satisfied by charm meson decay, the VS-limit nevertheless suggests that Cabibbo favored semileptonic $D$ decay will be dominated by the channels $D \rightarrow K l \nu$ and $D \rightarrow K^{*} l \nu$. Similarly, for Cabibbo disfavored decays, in which strong isospin is not conserved, the VS-limit predicts that $D^{0}$ decay should be dominated by $\pi l \nu$ and $\rho l \nu$, and $D^{+}$decay by $x l \nu, \eta l \nu$, $\eta^{\prime} l \nu, \rho l \nu$, and $\omega l \nu$. Searches either for modes involving higher mass resonances or for four- and five-body decays provide the most direct means of finding deviations from the VS-limit. The alternative technique, comparison of inclusive and summed 
exclusive decay rates, presently suffers from systematic uncertainties.

In the case of the $D^{0}$, four-prong decay vertices can have no contribution from the common Cabibbo favored and suppressed modes, and thus are predicted to be absent in the VS-limit. Using a sample of reconstructed four-prong semimuonic decay vertices ${ }^{10}$, E653 conducted a search for the decay modes $D^{0} \rightarrow K^{-} \pi^{+} \pi^{-} \mu^{+} \nu$ and $D^{0} \rightarrow\left(\bar{K}^{*}(892) \pi\right)^{-} \mu^{+} \nu$. The background in this sample was substantially reduced by using emulsion scanning ${ }^{b}$ to flag reconstruction errors. No evidence of a signal was found in either channel. By normalizing to the signal observed in $D^{0} \rightarrow K^{-} \mu^{+} \nu$, the following upper limits were determined at $90 \%$ confidence level

$$
\frac{\Gamma\left(D^{0} \rightarrow K^{-} \pi^{+} \pi^{-} \mu^{+} \nu\right)}{\Gamma\left(D^{0} \rightarrow K^{-} \mu^{+} \nu\right)}<0.037 \quad \text { and } \quad \frac{\Gamma\left(D^{0} \rightarrow\left(\bar{K}^{*}(892) \pi\right)^{-} \mu^{+} \nu\right)}{\Gamma\left(D^{0} \rightarrow K^{-} \mu^{+} \nu\right)}<0.043 \text {. }
$$

Applying invariance of strong isospin, and data from $\mathrm{PDG}^{11}$, gives the corresponding ratio for $D^{+}$decay (again at $90 \%$ C.L.)

$$
\frac{\Gamma\left(D^{+} \rightarrow \bar{K}^{*}(892)^{0} x^{0} \mu^{+} \nu\right)}{\Gamma\left(D^{+} \rightarrow \bar{K}^{*}(892)^{0} \mu^{+} \nu\right)}<3 \% \text {. }
$$

These results support the basic prediction of the VS-limit, and on a practical level indicate that multi-body or higher mass resonant modes are negligible in the study of three-body $D^{+}$and $D_{s}^{+}$semimuonic decay discussed in this review.

\section{Measurement of the mode $D^{+} \rightarrow \rho^{0} \mu^{+} \nu$}

As noted in the introduction, the Cabibbo suppressed semileptonic decay modes play an important role in determining ratios of CKM matrix elements. Only the $\pi l \nu$ modes have been previously observed ${ }^{7,12}$. By employing the $M_{\min }$ technique, E653 has recently reported a signal for the $D^{+} \rightarrow \rho(770)^{0} \mu^{+} \nu$ mode ${ }^{13}$. The $M_{\pi x}$ distribution for the final data sample is shown in Figure 3, and an enhancement of six events is clearly observed in the region of the $\rho^{0}$.

After subtracting all backgrounds, which were principally from $D_{s}^{+}$decay and from other Cabibbo disfavored $D^{+}$channels, the $D^{+} \rightarrow \rho^{0} \mu^{+} \nu$ signal was determined to be $4.0_{-2.3}^{+2.8} \pm 1.3$ events. (The statistical significance of the signal is more realistically reflected by the Poisson probability for the sum of all backgrounds to fluctuate up to or beyond the level of the final data set; that probability is $1.4 \%$.) Figure 3 also shows the sum of the simulated $D^{+} \rightarrow \rho^{0} \mu^{+} \nu$ signal and all backgrounds.

The ratio of $D^{+} \rightarrow \rho^{0} \mu^{+} \nu$ to $D^{+} \rightarrow \bar{K}^{* 0} \mu^{+} \nu$ was determined to be

$$
\frac{\Gamma\left(D^{+} \rightarrow \rho^{0} \mu^{+} \nu\right)}{\Gamma\left(D^{+} \rightarrow \bar{K}^{* 0} \mu^{+} \nu\right)}=0.044{ }_{-0.025}^{+0.031} \pm 0.014
$$

in agreement with the expectation that Cabibbo disfavored modes constitute a small fraction of the total semileptonic decay width.

bemulsion scanning was used in other results discussed in this review, but only to confirm background estimates. 


\section{Form Factors in $D^{+} \rightarrow \bar{K}^{* 0} \mu^{+} \nu$}

Measurement of the form factors in the $D^{+} \rightarrow \bar{K}^{* 0} \mu^{+} \nu$ decay mode is an important step in attempting to understand the discrepancy between the measured and predicted values of $\Gamma\left(D^{+} \rightarrow \bar{K}^{* 0} \mu^{+} \nu\right)$. Ratios of these form factors were determined by E653 from a sample of $305 D^{+} \rightarrow \bar{K}^{* 0} \mu^{+} \nu$ events ${ }^{14}$, in which the background contamination was less than $10 \%$ (see Figure $2 \mathrm{~b}$ ). The strong interaction effects in the decay can be described in terms of two axial vector form factors, $A_{1}\left(q^{2}\right)$ and $A_{2}\left(q^{2}\right)$, and one vector form factor, $V\left(q^{2}\right)$, where $q^{2}$ is the (mass) $)^{2}$ of the virtual $W^{+}$bosonc. Three decay angles are defined by convention: $\theta_{V}$ is the polar angle in the $\bar{K}^{* 0}$ rest frame between the $\pi^{+}$and the $D^{+}, \theta_{L}$ is the polar angle in the $W^{+}$rest frame between the $\nu$ and the $D^{+}$, and $\chi$ is the aximuthal angle in the $D^{+}$rest frame between the $\bar{K}^{* 0}$ and $W^{+}$decay planes. The form factor ratios at $q^{2}=0, A_{2}(0) / A_{1}(0)$ and $V(0) / A_{1}(0)$, were determined from a four-dimensional maximum likelihood fit to the data in $\cos \theta_{V}, \cos \theta_{L}, \chi$ and $q^{2} / q_{\max }^{2}$, where the maximum $q^{2}$ for a decay is given by: $q_{\max }^{2}=\left(M_{D}-M_{K \pi}\right)^{2}$. Figure 4 shows the uncorrected distributions for these decay variables.

The function fit was the full expression for the partial decay rate expressed in terms of the four decay variables ${ }^{15}$, and included the effects due to non-negligible muon mass ${ }^{16}$; the $q^{2}$-dependence of the form factors was assumed to be given by nearest pole dominance. The results of the fit were

$$
\frac{A_{2}(0)}{A_{1}(0)}=0.82_{-0.23}^{+0.22} \pm 0.11 \quad \text { and } \quad \frac{\mathrm{V}(0)}{\mathrm{A}_{1}(0)}=2.00_{-0.32}^{+0.34} \pm 0.16
$$

in agreement with a more recent measurement ${ }^{17}$. The distributions for simulated $D^{+} \rightarrow \bar{K}^{* 0}{ }^{+} \nu$ decays generated using the fit results are shown superimposed on the data in Figure 4.

Table 1. Comparison of E653 results to theoretical predictions for $D^{+} \rightarrow \bar{K}^{* 0} \mu^{+} \nu$ form factor ratios.

\begin{tabular}{|c|c|c|}
\hline & $A_{2}(0) / A_{1}(0)$ & $V(0) / A_{1}(0)$ \\
\hline E653 & $0.82_{-0.23}^{+0.22} \pm 0.11$ & $2.00_{-0.32}^{+0.34} \pm 0.16$ \\
\hline ISGW $^{8}$ & $1.0 \pm 0.3$ & $1.4 \pm 0.4$ \\
\hline $\mathrm{KS}^{15}$ & 0.8 & 0.8 \\
\hline $\mathrm{AW} / \mathrm{GS}^{18,19}$ & 0.8 & 1.9 \\
\hline $\mathrm{WSB}^{20}$ & 1.3 & 1.1 \\
\hline $\mathrm{BBD}^{21}$ & $1.2 \pm 0.2$ & $2.2 \pm 0.2$ \\
\hline $\operatorname{AOS}^{22}$ & $0.9 \pm 0.4$ & $1.9 \pm 0.8$ \\
\hline $\mathrm{BKS}^{23}$ & $0.70 \pm 0.16_{-0.15}^{+0.20}$ & $1.99 \pm 0.22_{-0.35}^{+0.31}$ \\
\hline
\end{tabular}

The ratios of form factors provide a stringent test of the predictive capability

\footnotetext{
$\mathrm{c}_{\mathrm{A}}$ third axial vector form factor, $A_{3}$, appears in the formalism weighted by the lepton mass squared; our data are insensitive to $A_{3}$.
} 
of the theoretical models. Table 1 compares the E653 measurements with quark models, QCD sum rules, and lattice QCD calculations; the WSB and KS quark models appear to be disfavored by the data at the level of $3 \sigma$.

\section{The Semimuonic Decays of the $D_{t}^{+}$}

The semimuonic decays of the $D_{t}^{+}$meson are of interest not only because they provide a further important testing ground for form factor models, but also because they play an important role in determining the absolute normalization of the $D_{t}^{+}$branching ratios. The technique for normalizing the branching ratios uses the predicted and measured values of the ratio $\Gamma\left(D_{a}^{+} \rightarrow \phi l \nu\right) / \Gamma\left(D^{+} \rightarrow \bar{K}^{*}(892)^{0} l \nu\right)$ and the measured values of $\Gamma\left(D^{+} \rightarrow \bar{K}^{*}(892)^{\circ} l \nu\right)$ and the $D^{+}$cross section to determine the $D_{t}^{+}$production cross section. All $D_{a}^{+}$branching ratios are thus normalized to the $D_{t}^{+} \rightarrow \phi l \nu$ mode, and hence the validity of this normalization technique rests on the accuracy of the model prediction for $\Gamma\left(D_{s}^{+} \rightarrow \phi l \nu\right) / \Gamma\left(D^{+} \rightarrow\right.$ $\bar{K}^{*}(892)^{0}(\nu)$. Measuring the form factors for $D_{s}^{+} \rightarrow \phi \mu^{+} \nu$ and relating them to those for $D^{+} \rightarrow \bar{K}^{*}(892)^{0} \mu^{+} \nu$ constitutes an important test of the model input.

E653 has conducted a study of the $D_{s}^{+} \rightarrow \phi \mu^{+} \nu$ mode analogous to its measurements of $D^{+} \rightarrow \bar{K}^{*}(892)^{0} \mu^{+} \nu$. The distribution in dikaon invariant mass, $M_{K K}$, for three-prong muonic vertices passing criteria optimized for selection of $D_{t}^{+} \rightarrow \phi \mu^{+} \nu$ is shown in Figure 5; a clear $\phi$ signal is apparent. For the events in this plot, both hadronic tracks have been assigned the kaon mass; hence there are contributions from $D^{+} \rightarrow \bar{K}^{* 0} \mu^{+} \nu$, which appears at $M_{K K}$ above the $\phi$ region, as well as from channels such as $D^{+}$or $D_{1}^{+}$to $\eta \mu^{+} \nu$ or $\eta^{\prime} \mu^{+} \nu$. Such potential backgrounds were reduced by requiring $M_{\min }\left(K K \mu^{+} \nu\right)$ (the minimum parent mass computed for a $K^{+} K^{-} \mu^{+} \nu$ final state) minus its error be less than $2.0 \mathrm{GeV} / \mathrm{c}^{2}$. Charm decay modes with unobserved neutral hadrons, such as $D_{a}^{+} \rightarrow \phi \mu^{+} \nu \pi^{0}$ and $D^{+} \rightarrow \phi \mu^{+} \nu \bar{K}^{0}$, are predicted to be small in analogy to the discussion of Section 4; nevertheless, any contamination was suppressed by demanding that $M_{\min }(K K \mu \nu)$ be greater than $1.6 \mathrm{Gev} / \mathrm{c}^{2}$. The mode $D^{+} \rightarrow \phi \mu \nu$ cannot occur via a spectator diagram, and is suppressed by the OZI rule.

After all cuts were applied, there were 24 events in the $\phi$ signal region, $1.013 \leq$ $M_{K K} \leq 1.026 \mathrm{GeV} / \mathrm{c}^{2}$; accounting for all backgrounds yielded $18.7 \pm 4.9_{-0.7}^{+0.4} D_{1}^{+} \rightarrow$ $\phi \mu^{+} \nu$ events ${ }^{24}$. Comparing this result to the signal for $D^{+} \rightarrow \bar{K}^{* 0}{ }_{\mu}{ }^{+} \nu$ reported ${ }^{5}$ by E653 yields the following ratio of cross sections times decay rates:

$$
\left(\frac{\sigma\left(D_{i}^{+}\right)}{\sigma\left(D^{+}\right)}\right)_{x_{F}>0} \cdot\left(\frac{\Gamma\left(D_{i}^{+} \rightarrow \phi \mu^{+} \nu\right)}{\Gamma\left(D^{+} \rightarrow \bar{K}^{* 0} \mu^{+} \nu\right)}\right)=0.56 \pm 0.14 \pm 0.12 .
$$

The ISGW2 quark potential model ${ }^{25}$ predicts that the ratio of decay rates in the above expression is 1.00; consequently, $\sigma\left(D_{t}^{+}\right) / \sigma\left(D^{+}\right)=0.56 \pm 0.14 \pm 0.12$, which is consistent with the results reported by previous experiments ${ }^{5}$.

The ratios of form factors for $D_{t}^{+} \rightarrow \phi \mu^{+} \nu, A_{2}(0) / A_{1}(0)$ and $V(0) / A_{1}(0)$, were determined in a similar fashion to that used for the $D^{+} \rightarrow \bar{K}^{* 0} \mu^{+} \nu$ mode, by 
fitting to the decay variables $\cos \theta_{V}, \cos \theta_{V}$ and $q^{2} / q_{\max }^{2}$. The results of the fit were

$$
\frac{A_{2}(0)}{A_{1}(0)}=2.1_{-0.5}^{+0.6} \pm 0.2 \quad \text { and } \quad \frac{V(0)}{A_{1}(0)}=2.3_{-0.9}^{+1.1} \pm 0.4
$$

The distributions for simulated events generated with these results are shown superimposed on the data in Figure 6.

The form factor ratios measured for $D_{a}^{+} \rightarrow \phi \mu^{+} \nu$ are compared to theoretical predictions in Table 2. The entry labelled "scaled ISGW2" is the result of multiplying the ISGW2 prediction for $D_{1}^{+} \rightarrow \phi \mu^{+} \nu$ by the ratio of the $\mathrm{E} 653$ result for $D^{+} \rightarrow \bar{K}^{* 0}{ }_{\mu}{ }^{+} \nu$ to the ISGW2 prediction for $D^{+} \rightarrow \bar{K}^{* 0}{ }_{\mu}+\nu$. The measured value of $A_{2}(0) / A_{1}(0)$ is $2.5 \sigma$ larger than the scaled ISGW2 prediction, which suggests that additional measurements are required before the model can be used with confidence in determining the normalization of the $D_{a}^{+}$branching ratios.

Table 2. Comparison of E653 results to theoretical predictions for $D_{a}^{+} \rightarrow \phi \mu^{+} \nu$ form factor ratios.

\begin{tabular}{lll} 
& $A_{2}(0) / A_{1}(0)$ & $V(0) / A_{1}(0)$ \\
\hline & & \\
E653 & $2.1_{-0.5}^{+0.6} \pm 0.2$ & $2.3_{-0.9}^{+1.1} \pm 0.4$ \\
Scaled ISGW2 & 0.83 & 2.31 \\
ISGW 2 $^{25}$ & 1.21 & 1.85 \\
BKs $^{23}$ & $0.78 \pm 0.08_{-0.13}^{+0.17}$ & $2.00 \pm 0.19$ \\
\end{tabular}

An additional test of the models involves relating the decay rate for $D_{t}^{+} \rightarrow$ $\phi \mu^{+} \nu$ to those for other $D_{s}^{+}$semimuonic channels. E653 has observed a signal ${ }^{24}$ for the decay chains $D_{0}^{+} \rightarrow \eta \mu^{+} \nu, \eta \rightarrow \pi^{+} \pi^{-} \pi^{0}$ or $\pi^{+} \pi^{-} \gamma$, and $D_{t}^{+} \rightarrow \eta^{\prime} \mu^{+} \nu$, $\eta^{\prime} \rightarrow \eta \pi^{+} \pi^{-}, \eta \rightarrow$ neutrals. Both chains give rise to three-prong muonic vertices and undetected neutral particles. The signal for $D_{t}^{+} \rightarrow\left(\eta+\eta^{\prime}\right) \mu^{+} \nu$, where $\left(\eta+\eta^{\prime}\right)$ indicates the sum of the $\eta$ and $\eta^{\prime}$ modes, is the enhancement in the dipion invariant mass, $M_{\pi x}$, between 0.3 and $0.4 \mathrm{GeV} / \mathrm{c}^{2}$ shown in Figure 7 . The distribution for simulated $D_{0}^{+} \rightarrow\left(\eta+\eta^{\prime}\right) \mu^{+} \nu$ events (generated with $\frac{2}{3} \eta$ and $\left.\frac{1}{3} \eta^{\prime}\right)$ is also shown in this figure. The data above $0.45 \mathrm{GeV} / \mathrm{c}^{2}$ is consistent with simulated $D^{+} \rightarrow$ $\bar{K}^{* 0} \mu^{+} \nu$ decays for which the kaon has been incorrectly assigned the pion mass. Requiring that $M_{K \pi}$ be less than $0.81 \mathrm{GeV} / \mathrm{c}^{2}$, and removing events with $M_{K K}$ in the $\phi$ signal region, strongly suppressed contributions from $D^{+} \rightarrow \bar{K}^{* 0} \mu^{+} \nu$ and $D_{t}^{+} \rightarrow \phi \mu^{+} \nu$, respectively.

After application of all cuts, 16 events remained with $M_{\pi \pi}$ in the $\left(\eta+\eta^{\prime}\right)$ signal region. Contamination remaining from $D^{+} \rightarrow \bar{K}^{* 0} \mu^{+} \nu$ and $D_{a}^{+} \rightarrow \phi \mu^{+} \nu$ decays was evaluated from the signals observed in these modes; that from Cabibbo disfavored semimuonic $D^{+}$channels was estimated from the ISGW2 model. Accounting for all backgrounds yielded $13 \pm 4$ events from $D_{a}^{+} \rightarrow\left(\eta+\eta^{\prime}\right) \mu^{+} \nu$. The Poisson 
probability that the background fluctuate up to or beyond the level of the final event sample was less than $10^{-6}$.

The signals observed for the two $D_{*}^{+}$decay modes yield the ratio

$$
\frac{\Gamma\left(D_{s}^{+} \rightarrow\left(\eta+\eta^{\prime}\right) \mu^{+} \nu\right)}{\Gamma\left(D_{d}^{+} \rightarrow \phi \mu^{+} \nu\right)}=3.9 \pm 1.6 \text {. }
$$

Scaling the prediction of the ISGW2 model for this quantity by the ratio of the measured value of $\Gamma(D \rightarrow K l \nu) / \Gamma\left(D \rightarrow K^{*} l \nu\right)$ (which equals $\left.1.49 \pm 0.24\right)^{6}$ to that predicted by the model should correct for some errors in the model. The scaled value of $\Gamma\left(D_{i}^{+} \rightarrow\left(\eta+\eta^{\prime}\right) \mu^{+} \nu\right) / \Gamma\left(D_{a}^{+} \rightarrow \phi \mu^{+} \nu\right)$ is 1.06 , or $1.8 \sigma$ below the E653 measurement. The disagreement, which is similar in magnitude to that observed in the form factor ratios, reinforces the conclusion that the model should be used with some caution until further measurements become available.

From a sample of five-prong muonic decays, E653 has also determined ${ }^{10}$

$$
\frac{\Gamma\left(D_{s}^{+} \rightarrow \eta^{\prime} \mu^{+} \nu\right)}{\Gamma\left(D_{s}^{+} \rightarrow \phi \mu^{+} \nu\right)}<1.6
$$

at $90 \%$ confidence level, suggesting that the $\eta^{\prime}$ mode does not dominate the $\left(\eta+\eta^{\prime}\right)$ semimuonic channels. This observation is in accord with the ISGW2 model, but is in contrast to the hadronic decay modes of the $D_{a}^{+}$, in which the $\eta^{\prime}$ modes appear to have larger branching fractions than the $\eta \operatorname{modes}^{26}$.

\section{Summary and Conclusions}

The E653 experiment has made a significant contribution to progress in understanding the semileptonic decay of charm mesons. Highlights have been the determination of form factor ratios in the decay $D^{+} \rightarrow \bar{K}^{* 0} \mu^{+} \nu$, the first observations of $D^{+} \rightarrow \rho^{0} \mu^{+} \nu$ and of $D_{s}^{+} \rightarrow\left(\eta+\eta^{\prime}\right) \mu^{+} \nu$, the first measurement of the $D_{i}^{+} \rightarrow \phi \mu^{+} \nu$ form factor ratios, and an improvement by a factor of four to the limit on four-body final states.

\section{Acknowledgements}

The work summarized in this paper depended on the efforts of all of our E653 colleagues, and on the help of the Fermilab staff in staging this experiment. We would like to thank $N$. Isgur for many informative discussions, and D. Scora for providing us with a preliminary version of his $\mathrm{PhD}$ thesis. 


\section{References}

1. M. Witherell, XVI International Symposium on Lepton-Photon Interactions, Ithaca, New York, August 1993.

2. N. Isgur and M. Wise, Phys. Lett. B232 (1989) 113, and B237 (1990) 447.

3. K. Kodama et al., Nuclear Instruments and Methods A289 (1990) 146.

4. K. Kodama et al., Phys. Rev. Lett. 66 (1991) 1819.

5. K. Kodama et al., Phys. Lett. B286 (1992) 187.

6. D. M. Potter, Proc. 1991 International Lepton-Photon Symposium and Europhysics Conf. on High Energy Physics, (World Scientific, 1992) 528.

7. J. Adler et al., Phys. Rev. Lett. 62 (1989) 1821.

8. N. Isgur, D. Scora, B. Grinstein and M. B. Wise, Phys. Rev. D39 (1989) 799, and D. Scora and N. Isgar, Phys. Rev. D40 (1989) 1491.

9. M. B. Voloshin and M. A. Shifman, Sov. J. Nucl. Phys 47(3) (1988) 511.

10. K. Kodama et al., Phys. Lett. B313 (1993) 260.

11. K. Hikasa et al, (Particle Data Group), Review of Particle Properties, Phys. Rev. D45 (1992) S1.

12. M. S. Alam et al., Phys. Rev. Lett. 71 (1993) 1311.

13. K. Kodama et al., Phys. Lett. B316 (1993) 455.

14. K. Kodama et al., Phys. Lett. B274 (1992) 246.

15. J. G. Korner and G. A. Schuler, Z. Phys. C38 (1988) 511.

16. J. G. Korner and G. A. Schuler, Z. Phys. C46 (1990) 93.

17. P. L. Frabetti et al., Phys. Lett. B307 (1993) 262.

18. T. Altomari and L. Wolfenstein, Phys. Rev. D37 (1988) 681.

19. F. J. Gilman and R. L. Singleton, Phys. Rev. D41 (1990) 142.

20. M. Wirbel, B. Stech, and M. Bauer, Z. Phys. C29 (1985) 627, and M. Bauer and M. Wirbel, Z. Phys. C42 (1989) 671.

21. P. Ball, V. M. Braun, and H. G. Dosch, Phys. Rev. D44 (1991) 3567.

22. T. M. Aliev, A. A. Ovchinnikov, and V. A. Slobodenyuk, Trieste preprint, IC/89/382.

23. C. W. Bernard, A. X. El-Khadra, and A. Soni, Phys. Rev. D45 (1992) 869.

24. K. Kodama et al., Phys. Lett. B309 (1993) 483.

25. D. Scora, PhD Thesis, University of Toronto (1992).

26. M. Daoudi et al., Phys. Rev. D45 (1992) 3965. 
FIGURE 1 :

Distributions of $M_{\min }$ for two-prong semimuonic candidate vertices and for charge two vertices (shaded histogram). The solid histogram corresponds to the simulation of all contributing $D^{0}$ decay modes.

FIGURE 2 :

a) Distribution of $M_{\min }$ for muonic three-prong secondary vertices. The solid histogram is the distribution for simulated $D^{+} \rightarrow \bar{K}^{* 0} \mu^{+} \nu, \bar{K}^{* 0} \rightarrow K^{-} x^{+}$decays, and the shaded distribution is for the wrong-sign vertices.

b) Distribution of $M_{K \pi}$ for vertices of Fig. $2 \mathrm{a}$ with $1.6<M_{\min }<2.0 \mathrm{GeV} / \mathrm{c}^{2}$. The distribution for the wrong-sign events is again shown shaded; the solid histogram corresponds to the fit described in the text. The final $M_{K \pi}$ cuts are indicated by vertical arrows.

\section{FIGURE 3 :}

Distribution of dipion invariant mass after application of cuts on all other variables. The data is the solid histogram; (the one event removed by an $M_{K \pi}$ mass cut used to eliminate the $\bar{K}^{*}(892)^{0}$ is hatched). The sum of simulated $D^{+} \rightarrow \rho^{0} \mu^{+} \nu$ decays and backgrounds from other charm decays is shown dashed. The arrows indicate the cuts used to define the $\rho^{0}$ mass region.

\section{FIGURE 4 :}

Raw $\cos \theta_{V}, \cos \theta_{L}, q^{2} / q_{\max }^{2}$ and $\chi$ distributions for the data. The dashed histograms are projections of the fit to $A_{2}(0) / A_{1}(0)$ and $V(0) / A_{1}(0)$.

\section{FIGURE 5 :}

Distribution of dikaon invariant mass (solid) for $D_{s}^{+} \rightarrow \phi \mu^{+} \nu$ candidates passing cuts on all other variables. Also shown (hatched) is the distribution for the wrongsign events, in which the muon has charge opposite to that of the vertex.

\section{FIGURE 6 :}

Raw $\cos \theta_{V}, \cos \theta_{L}$, and $q^{2} / q_{\max }^{2}$ distributions for the 24 events passing the $D_{s}^{+} \rightarrow \phi \mu^{+} \nu$ selection criteria. The dashed histograms are projections of the fit to $A_{2}(0) / A_{1}(0)$ and $V(0) / A_{1}(0)$. Efficiencies are shown as dot-dashed curves for each of the three variables.

\section{FIGURE 7 :}

Distribution of dipion invariant mass (solid) for $D_{*}^{+} \rightarrow\left(\eta+\eta^{\prime}\right) \mu^{+} \nu$ candidates passing initial selection criteria. Also shown are distributions for the wrong-sign background (hatched), for simulated $D_{s}^{+} \rightarrow\left(\eta+\eta^{\prime}\right) \mu^{+} \nu$ decays (dot-dashed curve) and for simulated $D^{+} \rightarrow \bar{K}^{* 0} \mu^{+} \nu$ decays (dashed curve). The arrow indicates the cut used to select the data. 


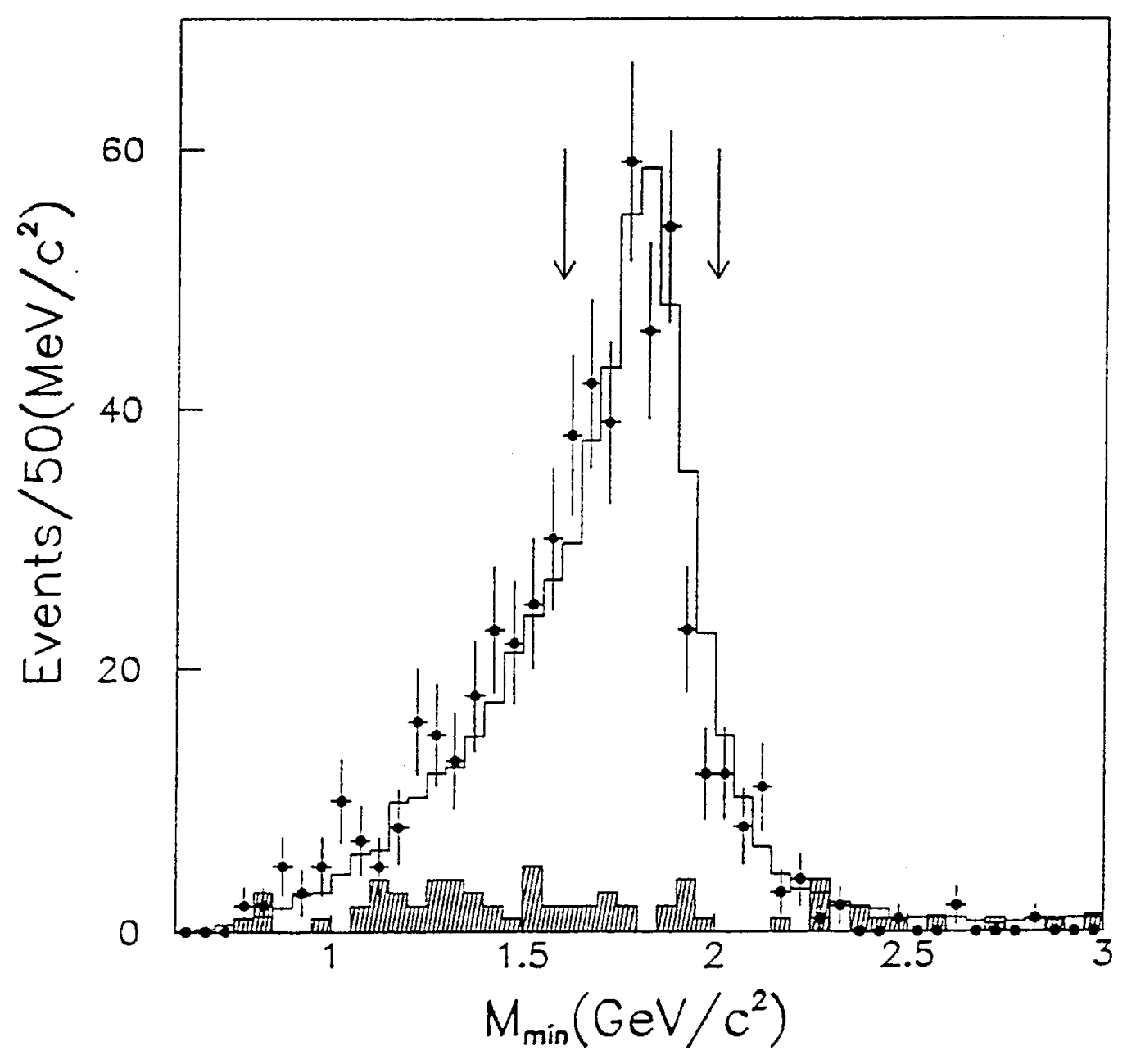

FIGURE 1 


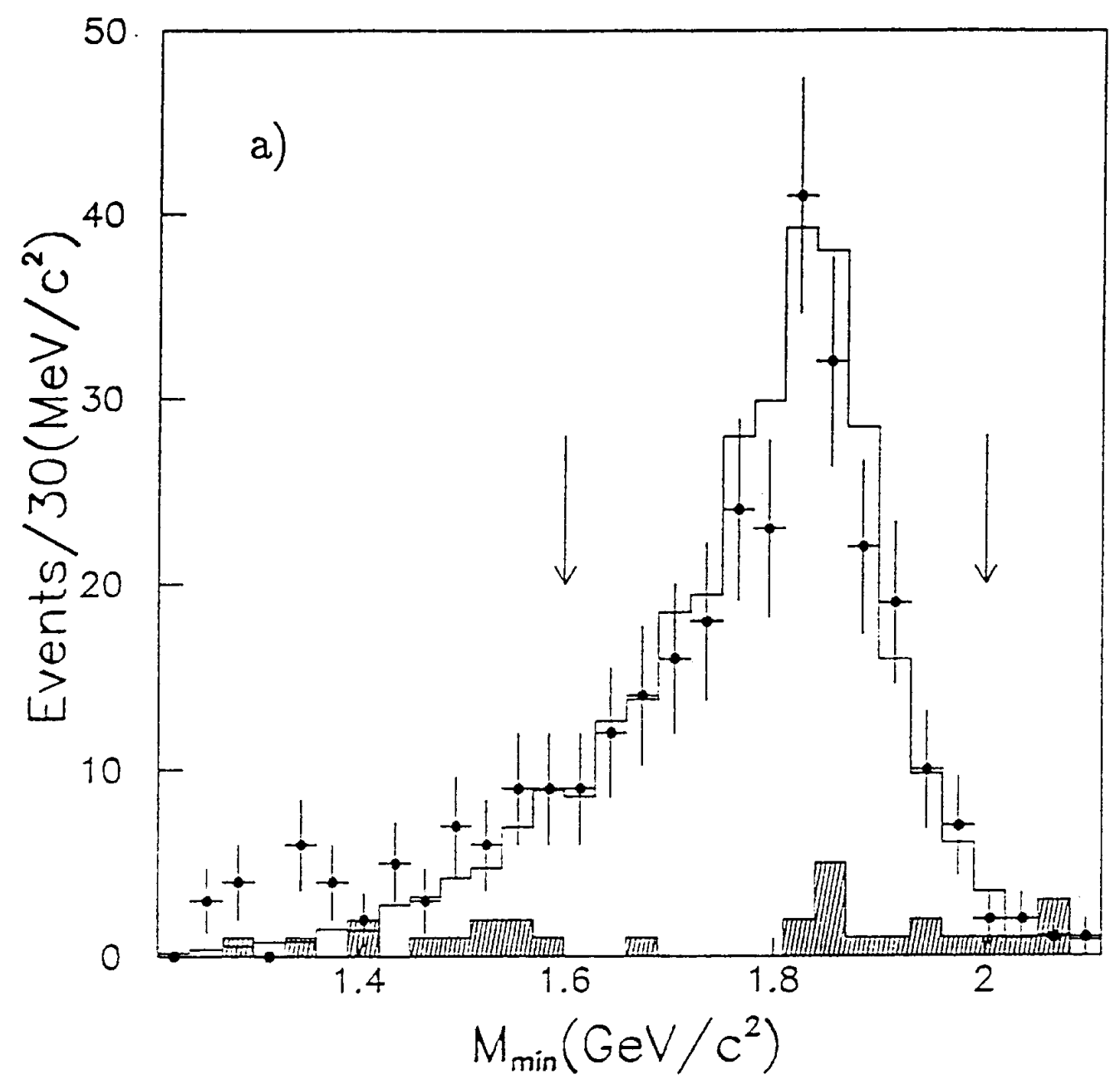

FIGURE 2a 


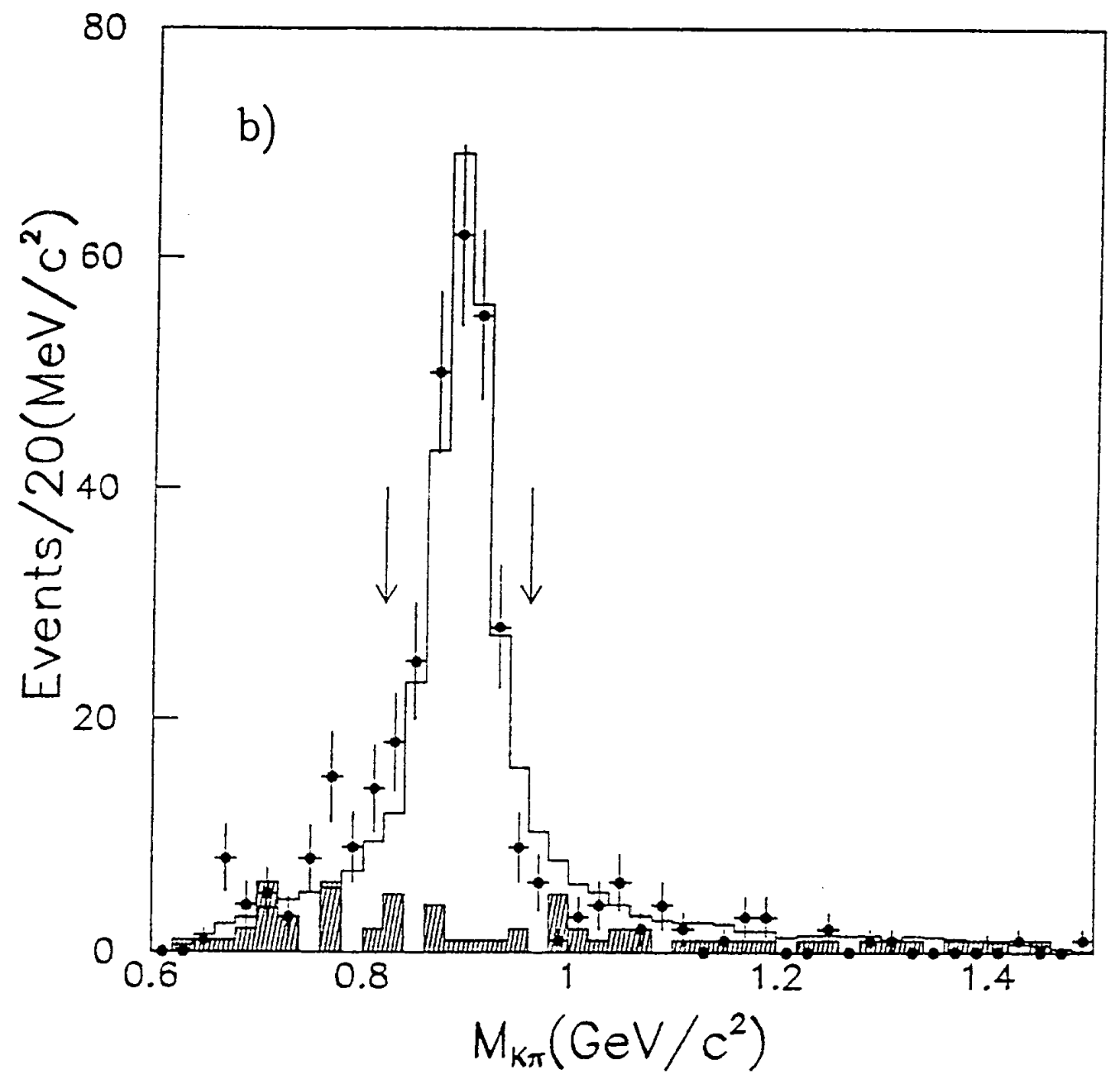

FIGURE 2b 


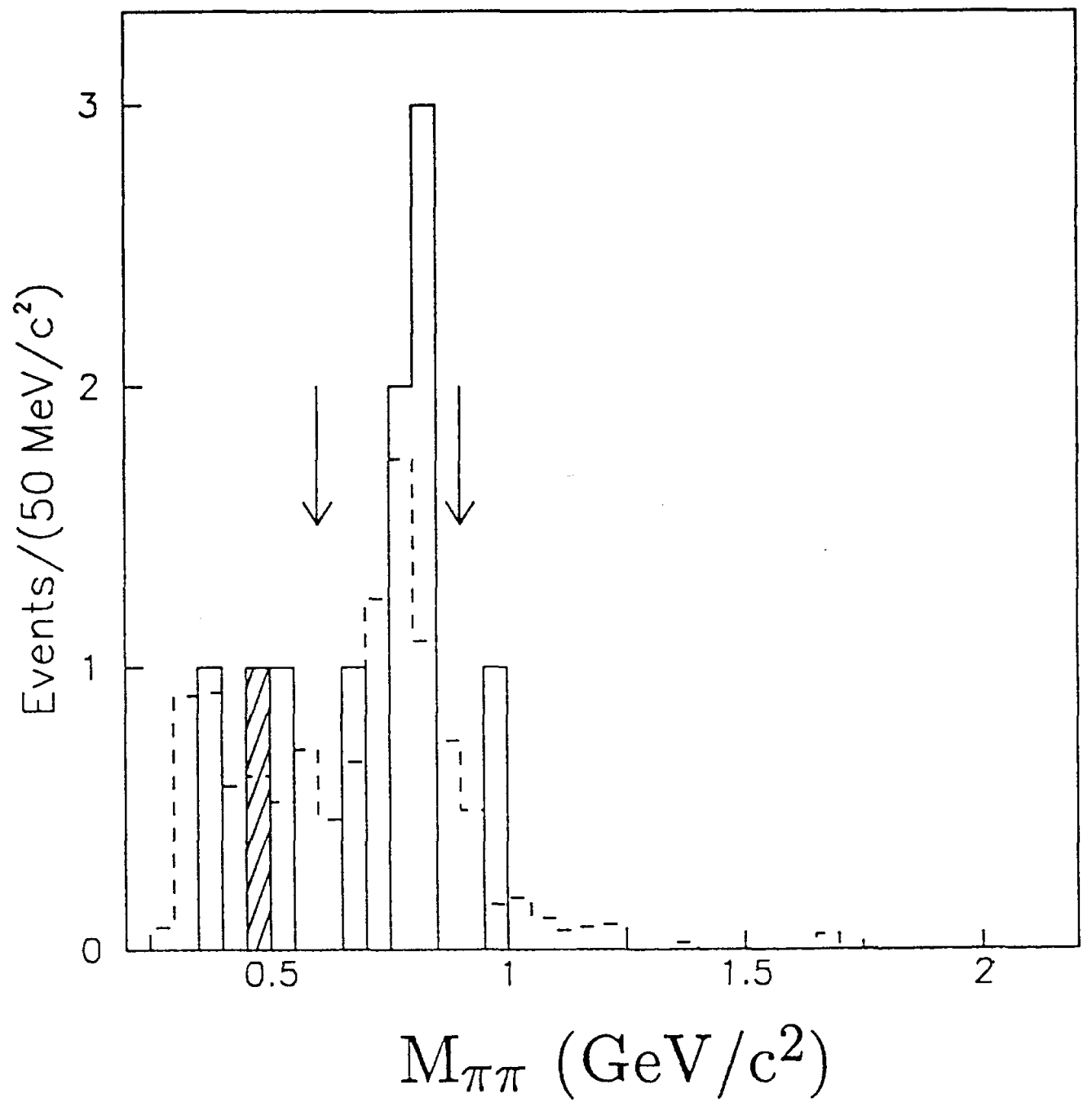

FIGURE 3 

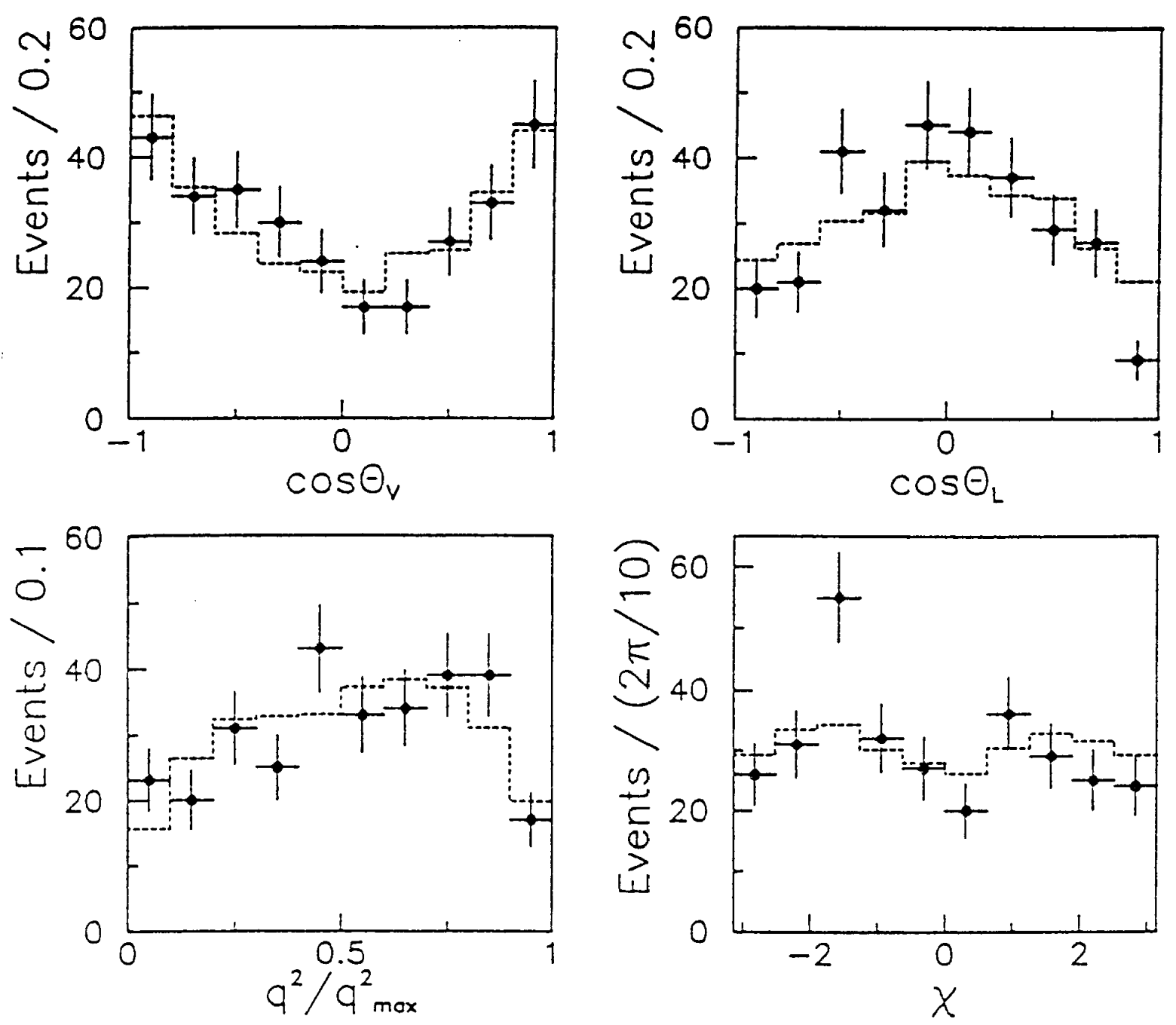

FIGURE 4 


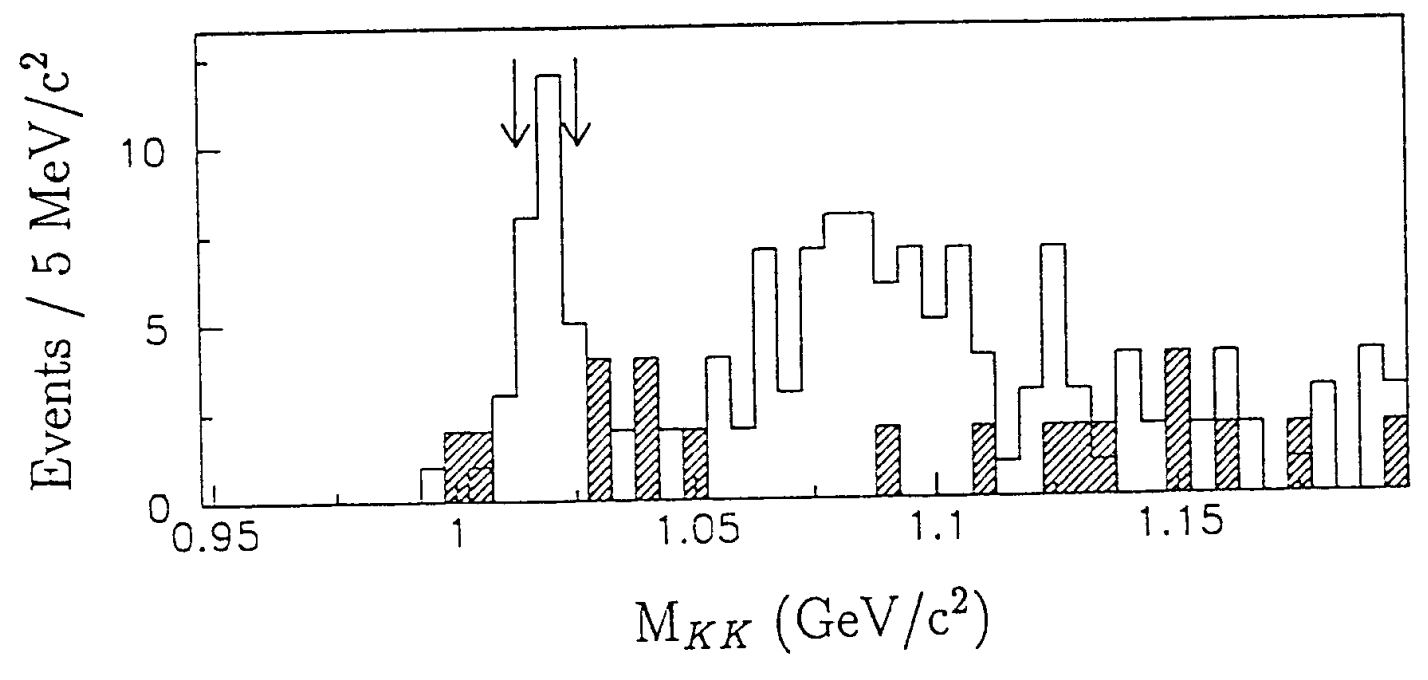

FIGURE 5 

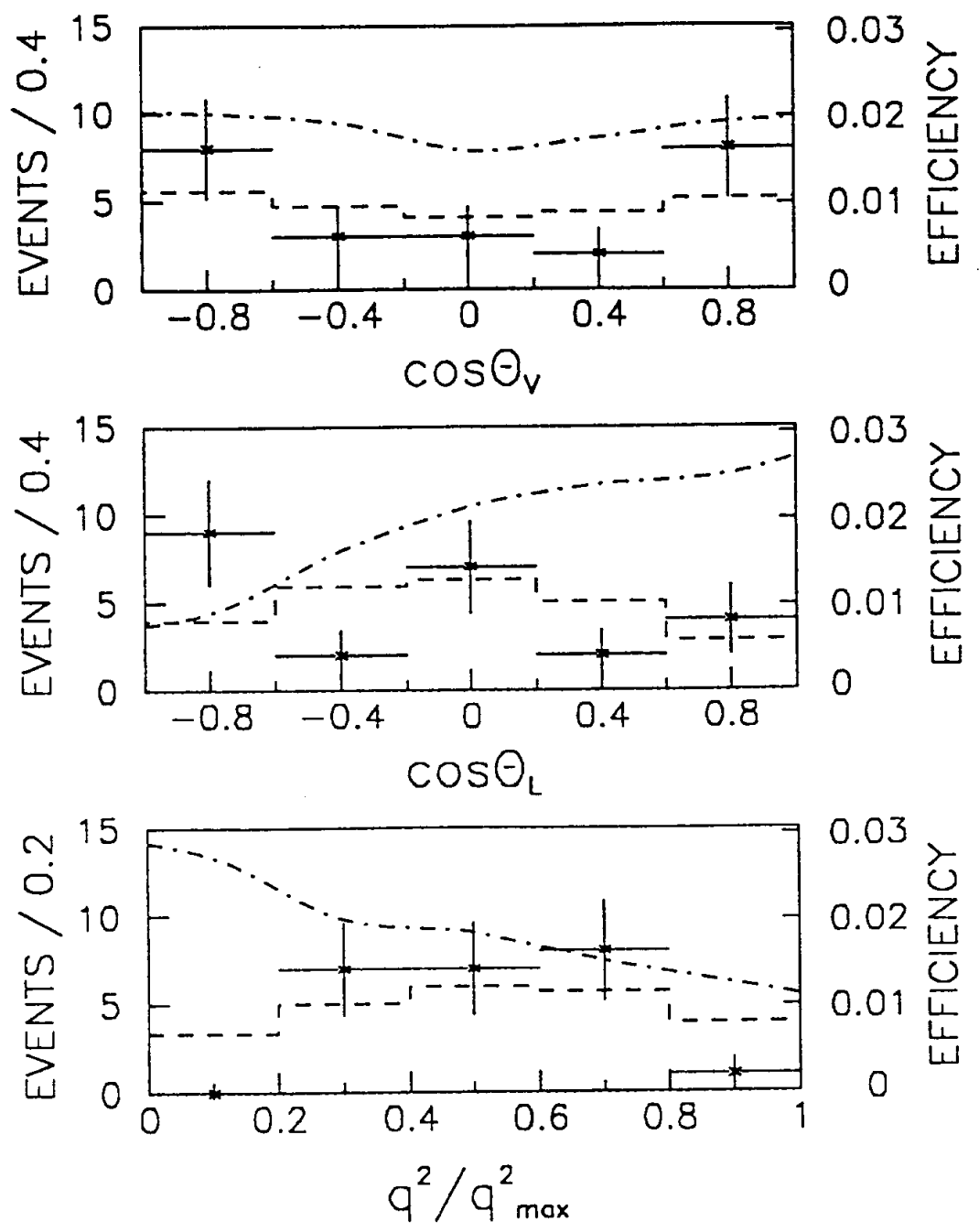

FIGURE 6 


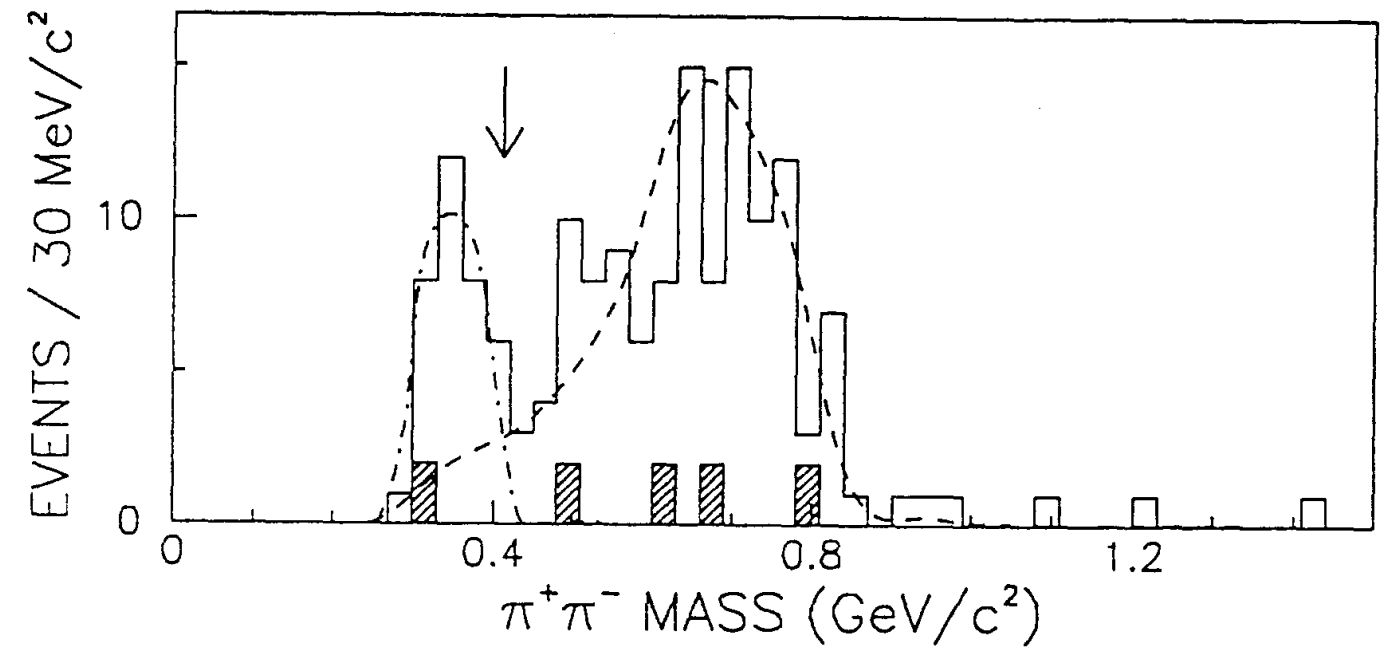

FIGURE 7 